\title{
ON THE INTEGRAL MEANS OF DERIVATIVES OF THE ATOMIC FUNCTION
}

\author{
MIODRAG MATELJEVIC AND MIROSLAV PAVLOVIC
}

\begin{abstract}
In this note we give upper and lower estimates on integral means of the atomic function and its derivatives over a circle of radius $r$ as $r$ approaches 1. From this we derive some known and new results.
\end{abstract}

1. Introduction. In 1971, M. R. Cullen [5] conjectured that $\phi^{\prime} \notin B^{1 / 2}$ for any singular inner function $\phi$. A counterexample was found by $\mathrm{H}$. A. Allen and C. L. Belna [3]; in fact, the atomic functions $S(z)=\exp [(z+1) /(z-1)]$ satisfies $S^{\prime} \in B^{p}$ for all $p<2 / 3$ and $S^{\prime} \notin B^{2 / 3}$. P. R. Ahern and D. N. Clark [2] generalized this and showed that $\phi^{\prime} \notin B^{2 / 3}$ provided that $\phi$ has a singular factor. Further references are [1 and 2].

Here we give good estimates of integral means of derivatives of $S(z)$ (our main result), and use these to find analogues of the above results for the spaces $D^{p}$ and $G^{p}$ (to be defined below).

2. Definitions. Let $f$ be an analytic function on the unit disc. We shall use the convenient notation,

$$
\begin{aligned}
& M_{p}(r, f)=\frac{1}{2 \pi} \int_{0}^{2 \pi}\left|f\left(r e^{i \theta}\right)\right|^{p} d \theta, \quad p>0, \\
& A(r, f)=\iint_{|z|<r}\left|f^{\prime}(z)\right|^{2} d x d y, \quad p>0 .
\end{aligned}
$$

The classes $D^{p}, A^{q, p}$ and $G^{p}$ are defined by $f \in D^{p}(p>0)$ if and only if $\int_{0}^{1} A(r, f)^{p / 2} d r<+\infty, f \in A^{q, p}(q>0,0<p<1)$ if and only if

$$
\int_{0}^{1} \int_{0}^{2 \pi}\left|f\left(r e^{i \theta}\right)\right|^{q}\left(1-r^{2}\right)^{1 / p-2} r d r d \theta<\infty
$$

$f \in G^{p}(p>0)$ if and only if $\int_{0}^{1}\left(\int_{0}^{2 \pi}\left|f^{\prime}\left(r e^{i \theta}\right)\right| d \theta\right)^{p} d r<\infty$.

The classes $B^{p}(0<p<1)$ is defined by $B^{p}=A^{1, p}$. For some properties of $D^{p}$ spaces see [6, 7, 9 and 10], for $G^{p}$ see [10 and 11].

Received by the editors November 10, 1981 .

1980 Mathematics Subject Classification. Primary 30A78.

Key words and phrases. Atomic function and its derivatives, integral means, singular functions. 
3. The results. The following theorem will be proven in $\S 4$.

THEOREM. Let $p>0$ and $n$ be a positive natural number. Then there is positive constant $K$ which depends only on $p$ and $n$ such that

$$
\frac{1}{K} \psi(r) \leqslant M_{p}\left(r, S^{(n)}\right) \leqslant K \psi(r), \quad r \rightarrow 1_{-},
$$

where $\psi(r)$ denotes $(1-r)^{1 / 2-n p}$ if $p>1 / 2 n,|\log (1-r)|$ if $p=1 / 2 n$ and 1 if $p<1 / 2 n$.

An immediate corollary is

Proposition 1. Let $n \in N$. Then

(i) $S^{(n)} \in D^{p}$ if and only if $p<4 /(4 n+1)$,

(ii) $S^{(n+1)} \in A^{q, p}$ if and only if $p<2 /(2 q(n+1)+1)$,

(iii) $S^{(n)} \in G^{p}$ if and only if $p<2 /(2 n+1)$.

If we set $n=1$ in part (i) and $n=0$ in (ii) of this proposition we obtain Theorems 5 and 3 in [8]. In particular, we have $S^{\prime} \notin B^{2 / 3}[4]$.

Proposition 1 implies $S^{\prime} \in D^{p}$ if and only if $p<4 / 5$ and $S^{\prime} \in G^{p}$ if and only if $p<2 / 3$. The following Propositions 2 and 3 generalize this fact.

Proposition 2. If $\phi$ is an inner function with a singular factor then (i) $\phi^{\prime} \notin D^{4 / 5}$ and (ii) $\phi^{\prime} \notin G^{2 / 3}$.

Proof. Let us consider (i). We have proved [9] $f \in D^{p}$ if and only if

$$
\sum_{0}^{\infty}\left(\sum_{k \in I_{n}} k^{1-2 / p}\left|a_{k}\right|^{2}\right)^{p / 2}<+\infty,
$$

where $f(z)=\sum a_{k} z^{k}$ and $I_{n}=\left\{k: 2^{n} \leqslant k<2^{n+1}, k \in N\right\}$. Hence, $D^{p} \subset A^{2, p / 2}$ for $0<p \leqslant 2$ and, in particular, $D^{4 / 5} \subset A^{2,2 / 5}$. Now (i) follows from Ahern's result [1] that $\phi^{\prime} \notin A^{2,2 / 5}$ if $\phi$ is an inner function with a singular factor.

Part (ii) follows from the relation $G^{p} \subset B^{p}, 0<p<1$ [10], and Ahern-Clark's result that $\phi^{\prime} \notin B^{2 / 3}$ if $\phi$ is an inner function with a singular factor.

We need the following definition [2]: a compact subset $E$ of $[0,2 \pi]$ is of type $\beta$ $(0<\beta \leqslant 1)$ if there is a constant $c$ such that $\left|E_{\varepsilon}\right| \leqslant c \varepsilon^{\beta}$, where $E_{\varepsilon}=\{\theta: \operatorname{dist}(\theta, E)$ $<\varepsilon\}$. It is clear that $E$ is finite if and only if $E$ is type 1 .

Proposition 3. Suppose $\sigma$ is a singular measure whose support is a set of type $\beta$ $(\beta>0)$. Let $\phi$ be the corresponding singular inner function. Then

(i) $\phi^{\prime} \in D^{p}$ for all $p<4 /(6-\beta)$,

(ii) $\phi^{\prime} \in G^{p}$ for all $p<2 /(4-\beta)$.

Proof. Let us first consider (i). Ahern [1, Lemmas 4.1 and 5.1] has proved

(2) $\phi^{\prime} \in B^{p}$ if and only if $\int_{0}^{1} M_{2}\left(r, \phi^{\prime}\right)(1-r)^{1 / p-1} d r<\infty \quad\left(\frac{1}{2}<p<1\right)$.

Hence

$$
\phi^{\prime} \in B^{p} \Rightarrow M_{2}\left(r, \phi^{\prime}\right)=O(1-r)^{-1 / p}, \quad r \rightarrow 1_{-}\left(\frac{1}{2}<p<1\right) .
$$

Combining (2) and (3) with Theorem 4 of [2], we get (i). 
For the proof of (ii) it is enough to note $M_{1}\left(r, \phi^{\prime}\right) \leqslant c(1-r)^{q-1}$ for all $q>\beta / 2$ (cf. [2, Theorem 4]).

4. The proof of the Theorem. The letter " $C$ " in the following should be read "an arbitrary constant, depending only on $p$ and $n$ ".

Let $p>0$ and $n$ a positive natural number. Induction gives

$$
S^{(n)}(z)=\frac{P_{n}(z)}{(z-1)^{2 n}} S(z),
$$

where $P_{n}$ is a polynomial and $P_{n}(1) \neq 0$. Hence, we obtain

(4) $\left|S^{(n)}\left(r e^{i \theta}\right)\right|=\frac{\left|P_{n}\left(r e^{i \theta}\right)\right|}{\left(1+r^{2}-2 r \cos \theta\right)^{n}} \exp \left(-\frac{1-r^{2}}{1+r^{2}-2 r \cos \theta}\right)$

$$
(0<r<1,0 \leqslant \theta \leqslant 2 \pi) \text {. }
$$

From (4) and the inequality $e^{-x} \geqslant 1-x$ it follows that

$$
\left|S^{(n)}\left(\mathrm{re}^{i \theta}\right)\right| \geqslant \frac{r\left|P_{n}\left(\mathrm{re}^{i \theta}\right)\right|}{2^{n}(1-r \cos \theta)^{n+1}}(r-\cos \theta) \quad(r>\cos \theta) .
$$

Since $P_{n}(1) \neq 0$, there are positive constants $C$ and $r_{0}, 0<r_{0}<1$, so that $\left|P_{n}\left(r e^{i \theta}\right)\right|$ $\geqslant C$ for all $(r, \theta)$ satisfying $0 \leqslant \theta \leqslant \pi / 2$ and $r_{0}^{2} \leqslant \cos \theta \leqslant r^{2}<1$. Hence,

$$
\left|S^{(n)}\left(r e^{i \theta}\right)\right| \geqslant C \frac{r-\cos \theta}{(1-r \cos \theta)^{n+1}} \quad\left(0 \leqslant \theta \leqslant \pi / 2, r_{0}^{2} \leqslant \cos \theta \leqslant r^{2}<1\right) .
$$

From this inequality, we obtain

$$
\begin{aligned}
M_{p}\left(r, S^{(n)}\right) & \geqslant C \int_{r_{0}^{2}}^{r^{2}} \frac{(r-u)^{p}}{(1-r u)^{n p+p}}(1-u)^{-1 / 2} d u \\
& \geqslant C \int_{r_{0}}^{r^{2}}(1-r u)^{-n p-1 / 2} d u \geqslant \frac{1}{K} \psi(r), \quad r \geqslant r_{0} .
\end{aligned}
$$

This establishes the left-hand inequality in (1).

For the rest, we note first that, by (4),

$$
M_{p}\left(r, S^{(n)}\right) \leqslant C+C \int_{0}^{\pi / 2}\left(1+r^{2}-2 r \cos \theta\right)^{-n p} \exp \left(-\frac{p\left(1-r^{2}\right)}{1+r^{2}-2 r \cos \theta}\right) d \theta,
$$

i.e.

$$
M_{p}\left(r, S^{(n)}\right) \leqslant C+C I_{1}(r)+C I_{2}(r)
$$

where

$$
\begin{aligned}
& I_{1}(r)=\int_{0}^{r} g(r, t)^{p}\left(1-t^{2}\right)^{-1 / 2} d t, \\
& I_{2}(r)=\int_{r}^{1} g(r, t)^{p}\left(1-t^{2}\right)^{-1 / 2} d t
\end{aligned}
$$

and

$$
g(r, t)=\left(1+r^{2}-2 r t\right)^{-n} \exp \left(-\frac{1-r^{2}}{1+r^{2}-2 r t}\right)
$$


Let $0 \leqslant t \leqslant r$. Then $1+r^{2}-2 r t \geqslant 1-r t$ and $1-t^{2}>1-r t$. Hence,

$$
I_{1}(r) \leqslant \int_{0}^{r}(1-r t)^{-n p-1 / 2} d t \leqslant C \psi(r), \quad r \rightarrow 1_{-} .
$$

To estimate $I_{2}(r)$ we use the equality (derived by direct calculation)

$$
\max _{r \leqslant t \leqslant 1} g(r, t)=n^{n}\left(1-r^{2}\right)^{-n} e^{-n} \quad\left(\frac{n-1}{n+1}<r<1\right) .
$$

From (7) it follows that

$$
I_{2}(r) \leqslant C(1-r)^{-n p} \int_{r}^{1}(1-t)^{-1 / 2} d t \leqslant C(1-r)^{-n p+1 / 2}, \quad r \rightarrow 1_{-} .
$$

Now the right-hand side of (1) follows immediately from (5), (6) and (8).

\section{REFERENCES}

1. P. R. Ahern, The mean modulus and derivative of an inner function, Indiana Univ. Math. J. 28 (1979), 311-347.

2. P. R. Ahern and D. N. Clark, On inner functions with $B^{p}$ derivative, Michigan Math. J. 23 (1976), 107-118.

3. H. A. Allen and C. L. Belna, Singular inner functions with derivative in $B^{p}$, Michigan Math. J. 19 (1972), 185-188.

4. C. L. Belna and B. Muckenhaupt, The derivative of the atomic function is not in $B^{2 / 3}$, Proc. Amer. Math. Soc. 63 (1977), 129-130.

5. M. R. Cullen, Derivatives of singular inner functions, Michigan Math. J. (1971), 283-287.

6. F. Holland and J. B. Twomey, On Hardy classes and the area functions, J. London Math. Soc. 17 (1978), 257-283.

7. Conditions for membership of Hardy spaces, Aspects of Contemporary Complex Analysis (D. A. Brannan and J. G. Clunie, eds.), Academic Press, New York, 1980, pp. 425-433.

8. M. Jevtić, Sur la dérivée de la fonction atomique, C. R. Acad. Sci. Paris 292 (1981), 201-203.

9. M. Mateljević and M. Pavlovic, $L^{p}$-behavior of power series with positive coefficients and Hardy space, Proc. Amer. Math. Soc. (to appear).

10. On weighted $L^{p}$ norms of area and length functions for analytic functions and Bergman spaces, Mat. Vesnik (to appear).

11. C. H. Pommerenke, Über die Mittlelwerte und Koeffizienten multivalenter Funktionen, Math. Ann. 145 (1962), 285-296.

Department of Mathematics, University of Belgrade, Studentski Trg 16, 11000 Belgrade, YUGOSLAVIA

Current address: Department of Mathematics, University of Wisconsin-Madison, Madison, Wisconsin 53706 